\title{
Na content dependence of superconductivity and the spin correlations in $\mathrm{Na}_{x} \mathrm{CoO}_{2} \cdot 1.3 \mathrm{H}_{2} \mathrm{O}$
}

\author{
Guo-qing Zheng ${ }^{1}$, Kazuaki Matano ${ }^{1}$, \\ R L Meng ${ }^{2}$, J Cmaidalka ${ }^{2}$ and C W $\mathbf{C h u}^{2,3,4}$ \\ ${ }^{1}$ Department of Physics, Okayama University, Okayama 700-8530, Japan \\ 2 Department of Physics and TCSAM, University of Houston, \\ TX 77204-5932, USA \\ ${ }^{3}$ Lawrence Berkeley National Laboratory, 1 Cyclotron Road, Berkeley, \\ CA 94720, USA \\ ${ }^{4}$ Hong Kong University of Science and Technology, Hong Kong, China \\ E-mail: zheng@psun.phys.okayama-u.ac.jp
}

\begin{abstract}
.
We report systematic measurements using ${ }^{59} \mathrm{Co}$ nuclear quadrupole resonance (NQR) technique on the cobalt oxide superconductors $\mathrm{Na}_{x} \mathrm{CoO}_{2} \cdot 1.3 \mathrm{H}_{2} \mathrm{O}$ over a wide $\mathrm{Na}$ content range $x=0.25 \sim 0.34$. We find that $T_{c}$ increases with decreasing $x$ but reaches to a plateau for $x \leq 0.28$. In the sample with $x \sim 0.26$, the spin-lattice relaxation rate $1 / T_{1}$ shows a $T^{3}$ variation below $T_{c}$ and down to $T \sim T_{c} / 6$, which unambiguously indicates the presence of line nodes in the superconducting (SC) gap function. However, for larger or smaller $x, 1 / T_{1}$ deviates from the $T^{3}$ variation below $T \sim 2 \mathrm{~K}$ even though the $T_{c}(\sim 4.7 \mathrm{~K})$ is similar, which suggests an unusual evolution of the SC state. In the normal state, the spin correlations at a finite wave vector become stronger upon decreasing $x$, and the density of states at the Fermi level increases with decreasing $x$, which can be understood in terms of a single-orbital picture suggested on the basis of LDA calculation.

PACS numbers: 74.25.Nf, 74.70.-b
\end{abstract}

\section{Introduction}

The layered cobalt oxides $\mathrm{Na}_{x} \mathrm{CoO}_{2}$ have attracted much attention in recent years, because of a rich variety of orders competing for the ground state. For $x=0.75$, the material shows a large thermoelectric power $[1,2]$, while the $x=0.5$ compound is a charge ordered insulator with $T_{C O}=50 \mathrm{~K}[3]$. The magnetic properties are different for different Na contents [3-5]. At the side with $x$ larger than 0.5 , the DC susceptibility shows a Curie-Weiss temperature $(T)$ variation, while at the low- $x$ side the susceptibility is $T$ independent [3]. Most interestingly, superconductivity with $T_{c} \sim 4.5 \mathrm{~K}$ emerges around $x=0.3$ when water molecules are intercalated in between the Co layers [6]. It has been reported that $T_{c}$ can be tuned by varying $x$ in the range of $0.25 \leq x \leq 0.38$ [7]. 
One of the unsettled issues on the superconductivity is the symmetry of the gap function. Theoretically, a chiral $(d+i d)$ superconducting state was first proposed on the basis of RVB scenario [8-10]. In a previous study, we found that the spin-lattice relaxation rate $1 / T_{1}$ shows no coherence peak below $T_{c}$, and follows a $T^{n}$ variation with $n=2.2 \sim 1$ at low $T$ [11]. This suggests that the superconductivity is of non-BCS type, but it has been pointed out that the data are insufficient to distinguish between some exotic states [12-14]. It has been shown that both chiral and pure $d$-wave states in the presence of disorder $[12,13]$, or an odd-frequency $s$-wave state [14] can be compatible with such $T$-variation of $T_{1}$. Since identification of the gap function is the first step toward the understanding of the superconductivity, more work is needed.

Another issue is how the electron correlations in the normal state evolve with doping, which should help understanding the mechanism for the superconductivity. In the octahedral crystal electric field environment, two $e_{g}$ orbitals of Co are located much higher in energy above $t_{2 g}$ orbitals. The local density approximation (LDA) band calculation shows that [15-17], among the three $t_{2 g}$ orbitals, the $a_{1 g}\left(3 d_{3 z^{2}-r^{2}}\right.$, where $z$-axis is the threefold rotation axis) orbit lies higher in energy than the remaining two $e_{g}$ orbitals. If one takes this picture $[8,9]$, then the $\mathrm{Co}^{4+}$ state without $\mathrm{Na}$ has one

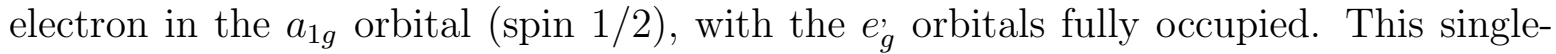
orbital situation resembles the undoped cuprates; adding $\mathrm{Na}$ dopes electrons into the $a_{1 g}$ orbital. In contrast, multiple-band models were also proposed [18,19], which draw similarities to the manganese oxides, another important class of strongly correlated materials.

In this letter, we address the above two issues by studying the evolution of the system with doping using NQR (nuclear quadrupole resonance) technique. We present intensive data on the superconducting and the normal states over a wide range of $\mathrm{Na}$ content.

\section{Experimental Results and Discussion}

The $\mathrm{Na}_{x} \mathrm{CoO}_{2} \cdot \mathrm{yH}_{2} \mathrm{O}$ powder [20] was synthesized following Ref. [6]. The precursor of $\mathrm{Na}_{0.75} \mathrm{CoO}_{2}$ was immersed in the $\mathrm{Br}_{2} / \mathrm{CH}_{3} \mathrm{CN}$ solution to de-intercalate Na. The amount of $\mathrm{Br}_{2}$ is listed in Table 1 , where $\mathrm{X}$ means the theoretical value necessary to remove all the $\mathrm{Na}$. The $\mathrm{Na}$ content of the final product was determined by the ion coupled plasma method. X-ray diffraction indicates that the samples are single phase except the 5X $(x \sim 0.34)$ and 10X $(x \sim 0.32)$ samples in which minor secondary phases are present. The c-axis elongates as the $\mathrm{Na}$ content decreases, in agreement with previous report [7]. $T_{c}$ was determined from the ac susceptibility and the $1 / T_{1}$ measurements. The previously reported sample [11] was made with $10 \mathrm{X} \mathrm{Br}$ amount in a separate run and turned out to have an $x \sim 0.31$. All other samples were made in the same run.

${ }^{59}$ Co NQR measurements were carried out by using a phase-coherent spectrometer. The nuclear magnetization decay curve is excellently fitted to the theoretical curves [24], with a unique $T_{1}$ component. 
Table 1. Characterizations of the samples.

\begin{tabular}{lllll}
\hline Br amount & Na content $x$ & c-axis length $(\AA)$ & a-axis length $(\AA)$ & $T_{c}(\mathrm{~K})$ \\
\hline $5 \mathrm{X}$ & 0.34 & 19.50 & 2.822 & 2.65 \\
\hline $10 \mathrm{X}$ & 0.32 & 19.62 & 2.822 & 3.60 \\
\hline $10 \mathrm{X}[11]$ & 0.31 & 19.59 & 2.819 & 3.70 \\
\hline $20 \mathrm{X}$ & 0.28 & 19.73 & 2.817 & 4.75 \\
\hline $40 \mathrm{X}$ & 0.25 & 19.80 & 2.817 & 4.70 \\
\hline $100 \mathrm{X}$ & 0.26 & 19.79 & 2.815 & 4.60 \\
\hline
\end{tabular}

Figure 1(a) shows a typical example of the three NQR transition lines arising from the nuclear spin $I=7 / 2$ of ${ }^{59}$ Co. Figure 1 (b) shows the $\pm 3 / 2 \leftrightarrow \pm 5 / 2$ transition line for various $\mathrm{Na}$ contents. Firstly, the peak frequency increases as $x$ decreases down to $x=0.28$. Analysis of three transition lines allows us to find that the $\nu_{Q}$ increases as the $\mathrm{Na}$ content decreases down to $x=0.28$, but the asymmetry parameter $\eta$ decreases monotonically with decreasing $\mathrm{Na}$ content, as seen in Fig. 1(c) and (d), respectively. Here $\nu_{Q}$ and $\eta$ are defined as $\nu_{Q} \equiv \nu_{z}=\frac{3}{2 I(2 I-1) h} e^{2} Q \frac{\partial^{2} V}{\partial z^{2}}, \eta=\left|\nu_{x}-\nu_{y}\right| / \nu_{z}$, with $Q$ being the nuclear quadrupole moment and $\frac{\partial^{2} V}{\partial \alpha^{2}}(\alpha=x, y, z)$ being the electric field gradient at the position of the nucleus [21]. The decrease of $\nu_{Q}$ with increasing $x$ is consistent with doping electrons into the $a_{1 g}$ orbit [23]. Secondly, for the $5 \mathrm{X}(x \sim 0.34)$ and 10X $(x \sim 0.32)$ samples, there appear multiple peaks for the transition. However, the additional peaks with smaller amplitude are not due to the minor impurity phase detected in the X-ray chart. Rather, we find that $1 / T_{1}$ measured in different peaks differs only by $10 \%$ in magnitude and shows the identical temperature variation; a clear decreases was seen below $T_{c}$. These peaks may have arisen from the ordering of $\mathrm{Na}$ ions [22] which are located above and below Co. More detailed discussion will be published elsewhere [23]. We note in passing that the multiple peaks seen in the \pm $3 / 2 \leftrightarrow \pm 5 / 2$ transition are not resolvable in the $\pm 5 / 2 \leftrightarrow \pm 7 / 2$ transition.

Figure 2 shows the temperature dependence of the quantity $1 / T_{1} T$ for various $x$. All measurements were done at the $\pm 3 / 2 \leftrightarrow \pm 5 / 2$ transition. For the $5 \mathrm{X}$ and $10 \mathrm{X}$ samples, $1 / T_{1}$ was measured at the main peak shown in Fig. 1(b). Two trends can be seen in Fig. 2. First, the absolute value increases with decreasing $x$. Second, $1 / T_{1} T$ increases upon lowering $T$, indicating the importance of spin correlations; the smaller the $x$ the steeper the increase, indicating that the spin correlations become stronger as $x$ is reduced.

What is the nature of the spin correlations? One good way to tell is through inspection of the Knight shift, which measures the spin susceptibility and, unlike the bulk susceptibility, is not affected by the presence of magnetic impurities. In a superconducting single crystal with $x \sim 0.3$, we found that the shift is $T$-invariant from $T_{c}$ up to $T=120 \mathrm{~K}$ [30], which is in good agreement with the report of Ning et al [31]. These results rule out the possibility of ferromagnetic spin fluctuations with wave vector $q=0$, since in that case the Knight shift would follow the temperature dependence of $1 / T_{1} T$ because both of them would be dominated by the susceptibility at wave vector 


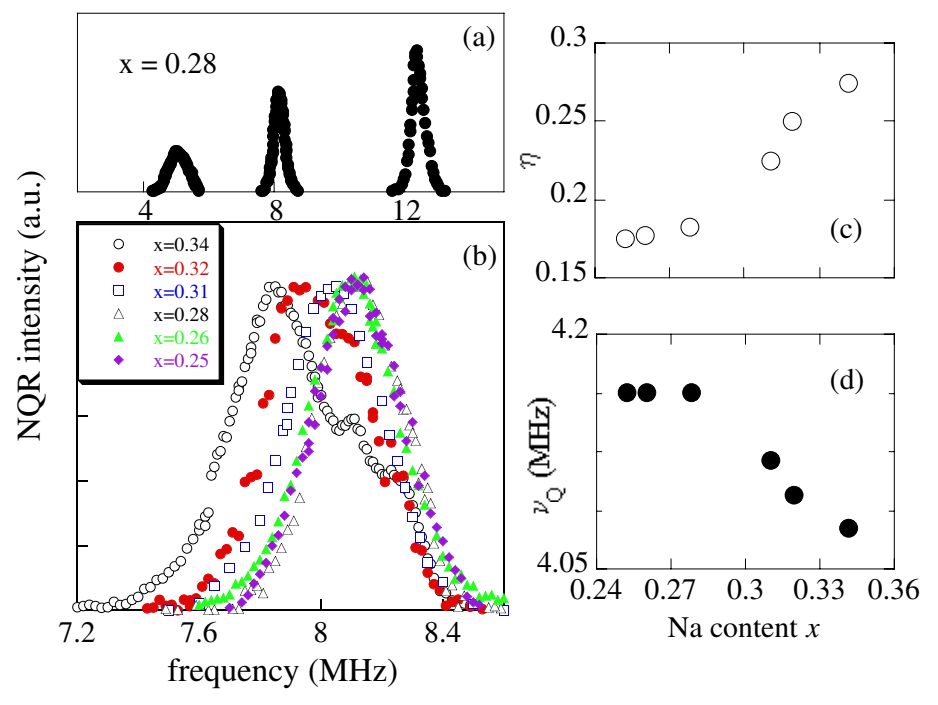

Figure 1. (a) ${ }^{59} \mathrm{Co} N \mathrm{NR}$ spectra for the sample of $x \sim 0.28$. (b) The line shape of the $\pm 3 / 2 \leftrightarrow \pm 5 / 2$ transition for various Na contents. (c) and (d) Na content dependencies of the asymmetric parameter $\eta$ and $\nu_{Q}$, respectively.

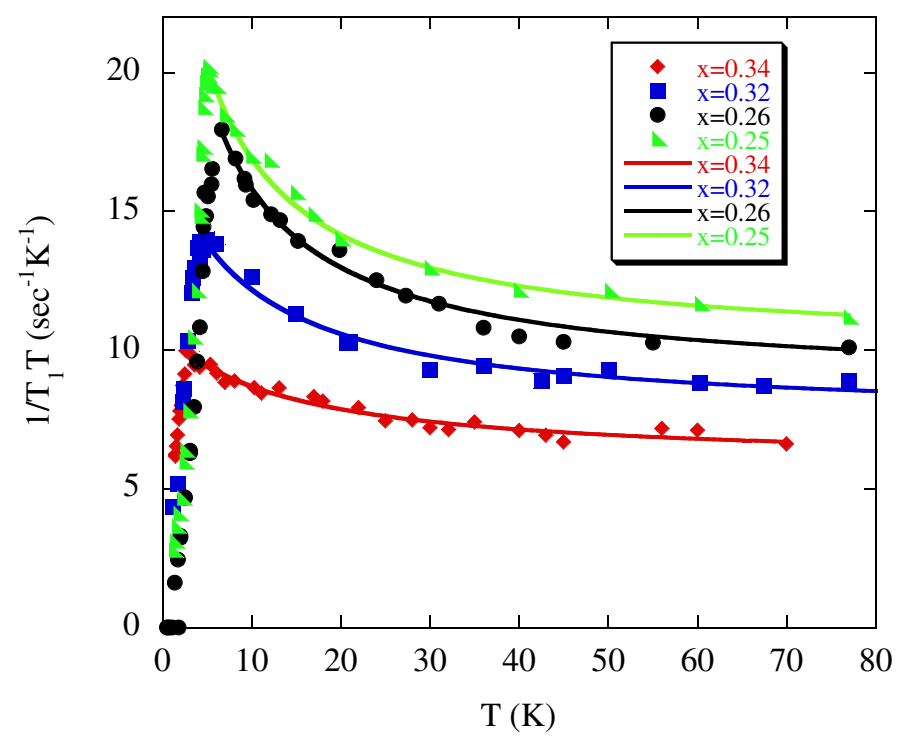

Figure 2. The quantity $1 / T_{1} T$ as a function of temperature for various Na contents. The curves are fits to $1 / T_{1} T=\left(1 / T_{1} T\right)_{0}+C /(T+\theta)$, with resulting parameter $C=75$, 77,111 and $123 \mathrm{Sec}^{-1}$ for $x=0.34,0.32,0.26$ and 0.25 , respectively. The values of $\left(1 / T_{1} T\right)_{0}$ and $\theta$ are plotted in Fig. 3 .

$q \sim 0$ 
The results thus indicate that the spin fluctuations peak at finite wave vector. The most likely candidate is antiferromagnetic (AF) correlations [8-10,32], although its detailed $q$-dependence awaits clarification by future experiments, in particular neutron scattering. Below we analyze our data in terms of AF correlations, in an attempt to quantify the discussion. In a general form, $1 / T_{1} T$ is written as $1 / T_{1} T=\sum_{q} A_{q}^{2} \chi^{\prime \prime}(q, \omega) / \omega$ in the $\omega \rightarrow 0$ limit, where $A_{q}$ is the hyperfine coupling constant, and $\chi "(q, \omega)$ is the imaginary part of the $q$-dependent, dynamical susceptibility. If one assumes that there is a peak around the $\mathrm{AF}$ wave vector $Q$, then one may have the following approximation: $1 / T_{1} T=\left(1 / T_{1} T\right)_{A F}+\left(1 / T_{1} T\right)_{0}$. Here $\left(1 / T_{1} T\right)_{0}$ denotes the contribution from wave vectors other than $Q$, which usually is dominated by the uniform susceptibility $\chi_{0}$. For the AF contribution, we use the formula for a two-dimensional nearly antiferromagnet, namely, $\left(1 / T_{1} T\right)_{A F}=\frac{C}{T+\theta}$ (Ref. [33]). We find that such model fits the data quite successfully, as depicted by the curves in Fig. 2. The resulting fitting parameters are shown in Figure 3. Note that a small value of $\theta$ means that a system is close to the AF instability $(\theta=0)$. The enhancement of the spin correlation (decrease of $\theta$ ) with decreasing $x$, as is the case in doped copper oxide high- $T_{c}$ superconductors, can be naturally understood in the single-orbital picture $[8,9]$ described earlier. In contrast, the multiple-band theory predicts $\mathrm{Na}$ content-insensitive, ferromagnetic spin correlations [19].

Turning to the quantity $\left(1 / T_{1} T\right)_{0}$, its increase with decreasing $x$ can be attributed to the increase of density of state (DOS) at the Fermi level. This result is consistent with the LDA band calculation which found that the dominant Fermi surface is a hole-like one and that the DOS increases as the electron number is reduced [16,34]. This aspect is different from the case of doped cuprates where the DOS decreases as decreasing carrier density. We summarize our results in Fig. 3. As $x$ decreases from $0.34, T_{c}$ increases, but reaches to a plateau of $\sim 4.7 \mathrm{~K}$ for $x \leq 0.28$. Our results for high value of $x$ are in good agreement with the report by Schaak et al [7]. A plateau has also been reported recently by Chen et al and Milne et al $[25,26]$. It is interesting to note that as the parameter $\theta$ decreases and $C$ increases, namely, as the electron correlation becomes stronger, $T_{c}$ increases.

Next, we turn to the superconducting state. As can be seen already in Fig. 2, no coherence peak was seen just below $T_{c}$ in all samples, irrespective of $\mathrm{Na}$ content and/or $T_{c}$ value. However, the low- $T$ behavior is sample dependent. We first show in Fig. 4 the results for the sample with $x \sim 0.26$. It can be seen that $1 / T_{1}$ follows a $T^{3}$ variation down to the lowest temperature measured $\left(\sim T_{c} / 6\right)$. This result indicates unambiguously that there exists line-nodes in the gap function. As we noted in previous publications $[11,27]$, the gap function with line nodes generates an energy $(E)$-linear DOS at low $E$ which results in a $T^{n}(n=3)$ dependence of $1 / T_{1}$. In the previous sample, $1 / T_{1}$ did not follow an exact $T^{3}$ variation and became proportional to $T$ below $T \sim T_{c} / 2$, [11]. It has been proposed that many exotic superconducting states can be compatible with the data [12-14]. Calculations incorporating impurity/disorder scattering showed that a $d_{x^{2}-y^{2}}+\mathrm{i} d_{x y}$ state which has a full gap is more consistent with the previous data [12]. 


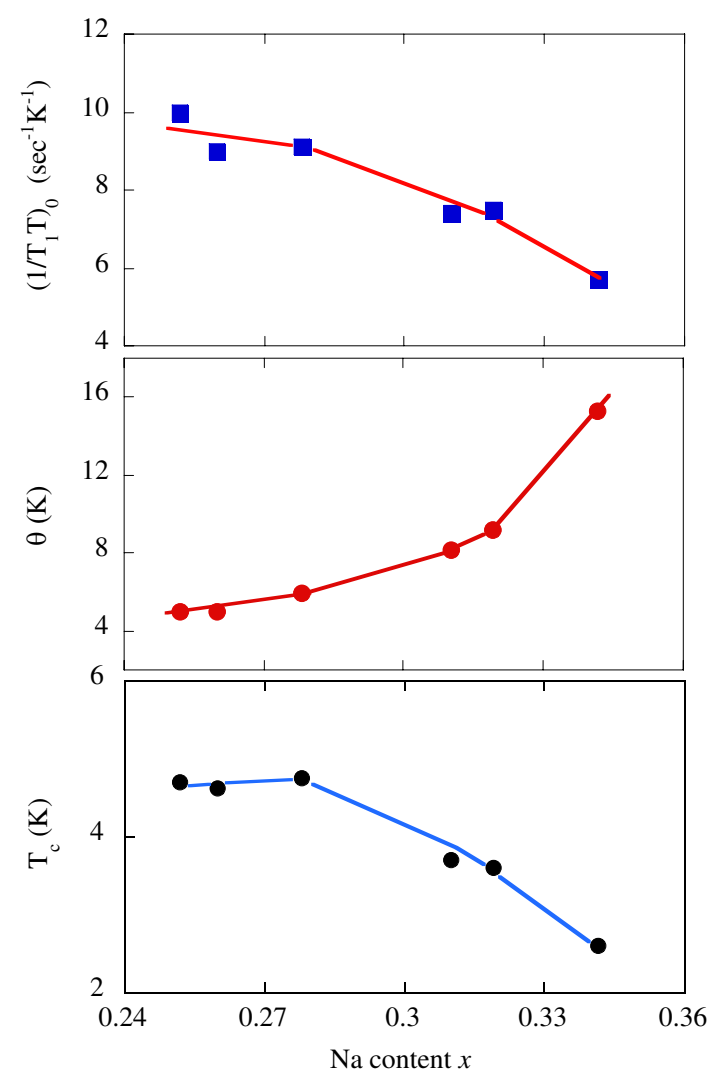

Figure 3. Various parameters obtained in this study plotted as a function of $\mathrm{Na}$ content, $x$. The curves are guides to the eyes.

Our present result completely rules out the $d_{x^{2}-y^{2}}+\mathrm{i} d_{x y}$ state proposed by the $t-J$ model in an isotropic triangular lattice [8-10,28]. Also, the odd frequency state proposed [14] is gapless [35,36], and is incompatible with our finding in the $x=0.26$ sample. However, if anisotropy of the $t$ term is incorporated, the theories could be compatible with our experimental results. In fact, it has been recently shown that a small anisotropy in $t$ (10 20\%) lifts the degeneracy between $d_{x^{2}-y^{2}}$ and $d_{x y}$ states, leading to a stabilization of a pure $d$-wave state [29].

In other samples with either larger or smaller $x$, however, $1 / T_{1}$ deviates from the $T^{3}$ relation below $T=2.0 \mathrm{~K}$. To see this more clearly, we plot the $T$-dependence of $1 / T_{1}$ for all samples in Fig. 5. In the inset of the figure is shown the normalized $1 / T_{1}$ by its value at $T=T_{c}$ against the reduced temperature. What is responsible for the deviation from the $T^{3}$ variation in samples with $x$ either larger or smaller than 0.26? Disorder or impurity is an unlikely candidate, since the samples with $x \sim 0.28$ and $x \sim 0.25$ have very similar or even higher $T_{c}$ and NQR line-width as the $x \sim 0.26$ sample. Clearly, this unprecedented property deserves further investigation, both experimentally and theoretically. Meanwhile, low-lying excitations due to nearby competing orders may be a possible candidate. For larger $x$, it is known that charge ordering [3,22] occurs. The Na 


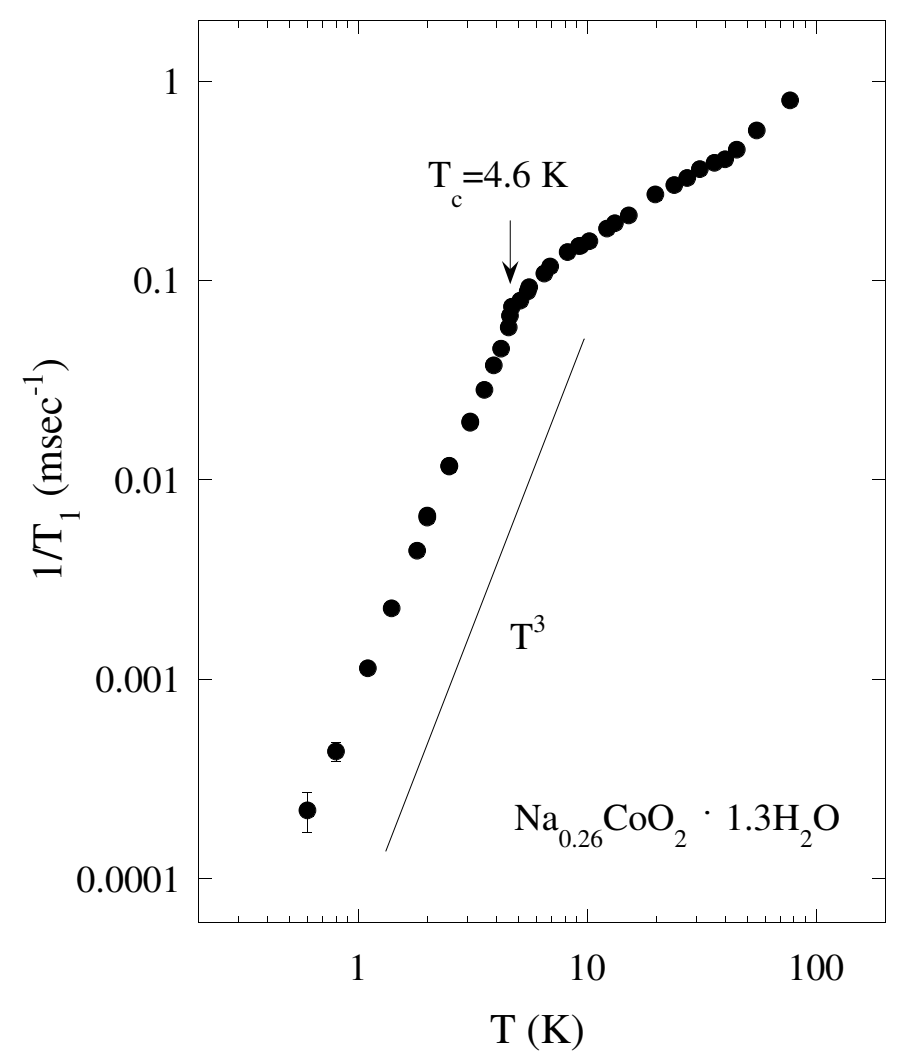

Figure 4. Temperature dependence of $1 / T_{1}$ for the sample of $x=0.26$. The arrow indicates $T_{c}$. The straight line depicts the $T^{3}$ variation.

order could also give rise to the anisotropy of the hopping integral $t$ mentioned earlier. For smaller $x$, on the other hand, it has been proposed that different charge/magnetic order may occur at $x=1 / 4[37]$.

\section{Conclusion}

In conclusion, we have presented intensive ${ }^{59} \mathrm{Co}-\mathrm{NQR}$ measurements on the cobalt oxide superconductors $\mathrm{Na}_{x} \mathrm{CoO}_{2} \cdot 1.3 \mathrm{H}_{2} \mathrm{O}$ over a wide $\mathrm{Na}$ content range $x=0.25 \sim 0.34$. We find that $T_{c}$ increases with decreasing $x$ but reaches to a plateau for $x \leq 0.28$. In the superconducting state, the spin-lattice relaxation rate $1 / T_{1}$ shows a $T^{3}$ variation down to $T \sim T_{c} / 6$ for $x \sim 0.26$, which indicates unambiguously the presence of line nodes in the superconducting gap function. For both larger or smaller $x$, however, $1 / T_{1}$ deviates from the $T^{3}$ dependence even through the $T_{c}$ value is similar. This unprecedented finding shows the richness of the physics of this new class of superconductors, and any pertinent theory must explain this peculiar property. In the normal state, the temperature dependence of $1 / T_{1} T$ indicates that the spin correlations at finite wavevector become stronger upon decreasing $x$, while the DOS increases. This aspect is 


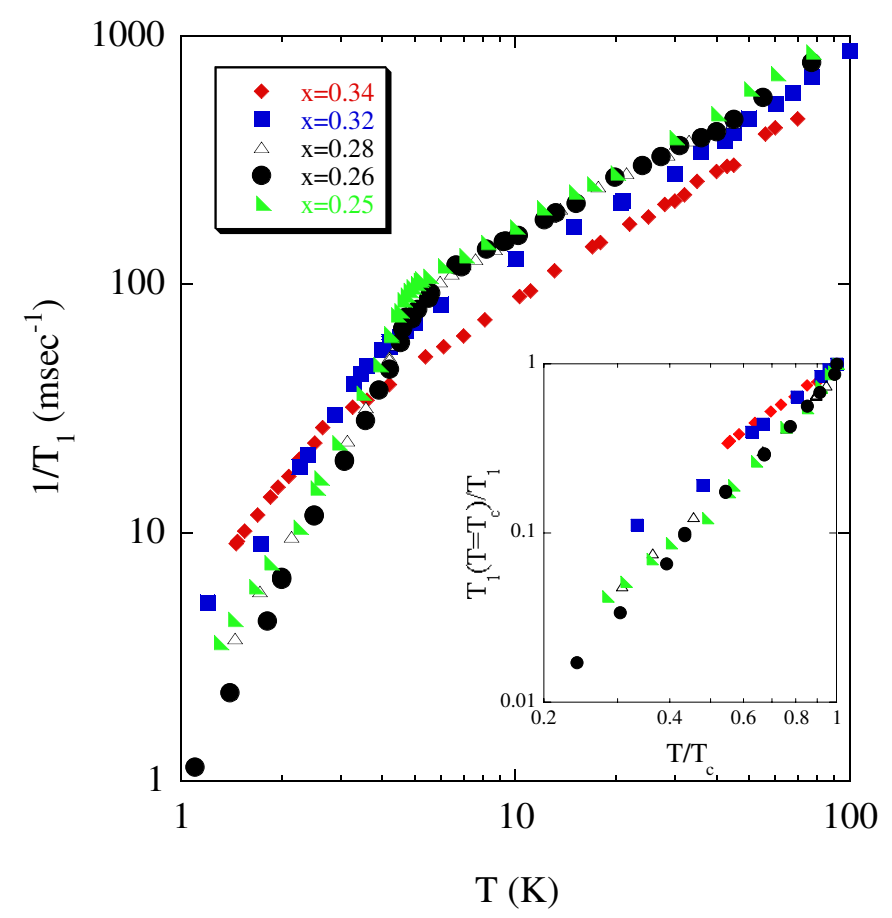

Figure 5. A comparison of the temperature dependence of $1 / T_{1}$ in the superconducting state for various samples with different Na content. The inset shows the normalized $1 / T_{1}$ by its value at $T=T_{c}$ against the reduced temperature.

different from the case of doped cuprates but can be understood in terms of a singleorbital picture suggested on the basis of LDA calculation.

\section{Acknowledgments}

We thank Y. Sun for x-ray chart analysis, G. Baskaran, Y. Fuseya, H. Harima, Y. Kitaoka, G.Khaliullin, C.T. Lin, K. Miyake, M. Ogata and Y. Yanase for helpful discussions. This work was supported in part by research grants from MEXT, NSF, the T. L. L. Temple Foundation, the John and Rebecca Moores Endowment, and DoE.

\section{References}

[1] I. Terasaki, Y. Sasago and K. Uchinokura, Phys. Rev. B 56, R12685 (1997).

[2] Yayu Wang, N. Rogado, R.J. Cava and N.P. Ong, Nature 423, 425 (2003).

[3] M. L. Foo et al, Phys. Rev. Lett. 92, 247001 (2004).

[4] C. Barnhard et al, Phys. Rev. Lett. 93, 167003 (2004).

[5] A. T. Boothroyd et al, Phys. Rev. Lett. 92, 197201 (2004).

[6] K. Takada et al, Nature 422, 53 (2003).

[7] R.E. Schaak et al, Nature 424, 527 (2003). 
[8] G. Baskaran, Phys. Rev. Lett. 91, 097003 (2003).

[9] Wang, Q.-H., D.-H. Lee and P.A. Lee, Phys. Rev. B 69, 092504 (2004).

[10] M. Ogata, J. Phys. Soc. Jpn. 72, 1839 (2003).

[11] T. Fujimoto, G. - q. Zheng, Y. Kitaoka, R.L. Meng, J. Cmaidalka and C.W. Chu, Phys. Rev. Lett. 92, 047004 (2004).

[12] Y. Bang et al, Phys. Rev. B 68, 212504 (2003)

[13] Q-H Wang and Z.D. Wang, Phys. Rev. B 69, 092502 (2004).

[14] M.D. Johnees et al, Phys. Rev. Lett.93, 097005 (2004).

[15] D. J. Singh, Phys. Rev. B61, 13397 (2000).

[16] L. J. Zou, J.L. Wang and Z. Zeng, Phys. Rev. B69, 132505 (2004).

[17] J. Kunes, K.W. Lee and W.E. Pickett, Phys. Rev. B 70, 045104 (2004) .

[18] G.Khaliullin, W.Koshibae, and S.Maekawa, Phys. Rev. Lett. 93,176401 (2004).

[19] Y. Yanase, M. Mochizuki and M. Ogata, J. Phys. Soc. Jpn. 74, 430 (2005).

[20] B. Lorenz et al, Phys. Rev. B 68, 132504 (2003).

[21] A. Abragam, The Principles of Nuclear Magnetism (Oxford Univ. Press, London, 1961).

[22] H. W. Zandbergenet al, Phs. Rev. B 70, 024101 (2004).

[23] K. Matano and G. - q. Zheng, in preparation.

[24] J. Chepin and J. H. Ross, J. Phys. Condens. Matt. 3, 8103 (1991).

[25] D. P. Chen et al, Phys. Rev. B 70, 024506 (2004).

[26] C.J. Milne et al, Phys. Rev. Lett. 93, 247007 (2004).

[27] G. - q. Zheng et al, Phys. Rev. Lett. 86, 4664 (2001).

[28] B. Liu, Y. Liang and S. Feng, Commun. Theor. Phys. 43, 1127 (2005).

[29] T. Watanabe, H. Yokoyama, Y. Tanaka, J. Inoue and M. Ogata, cond-mat/0411711.

[30] The Knight shift contains substantial contribution from the spin susceptibility (G. - q. Zheng, K. Matano, D. P. Chen and C. T. Lin, to be published).

[31] F.L. Ning et al, Phys. Rev. Lett. 93, 237201 (2004).

[32] G. Gasparovic et al, cond-mat/0508158

[33] T. Moriya, K. Ueda, Y. Takahashi, J. Phys. Soc. Jpn. 59, 2905 (1990).

[34] P. Zhang, W. Luo, V. Crespi, M. L. Cohen, and S. Louie1, Phys. Rev. B 70, 085108 (2004).

[35] I.I. Mazin and M.D. Johannes, cond-mat/0506536

[36] Y. Fuseya, H. Kohno and K. Miyake, J. Phys. Soc. Jpn, 72, 2914 (2003).

[37] G. Baskaran, cond-mat/0306569 